\title{
Ralf Bartenschlager, Charles Rice, and Michael Sofia are honored with the 2016 Lasker DeBakey Clinical Medical Research Award
}

$\mathbf{H}$ and causes hepatitis that ranges from a mild disease that is cleared without intervention to a life-long chronic disease that causes extreme damage to the liver. It is currently estimated that 130-150 million people throughout the world are infected with $\mathrm{HCV}$ and a substantial number of these individuals will develop cirrhosis and/or liver cancer. Unfortunately, many infected individuals remain asymptomatic for years after the initial infection and may not be diagnosed until the onset of symptoms associated with severe liver damage (1).

HCV was first identified in 1989 as the causative agent of a transfusion-associated, non-A, non-B hepatitis (NANBH) that often progressed to cirrhosis and subsequent liver cancer. With the identification of the virus, researchers moved toward the development of cell culture models to allow evaluation of the HCV life cycle and identification of possible drug targets. This would not be an easy task, as HCV proved to be less than amenable to techniques that had previously been successful for establishing in vitro and small animal models of other viral infections. Through the work of several dedicated researchers, cell culture models for HCV came to fruition, allowing a better understanding of the viral life cycle and the involved host factors as well as paving the way for drug development and testing. The 2016 Lasker DeBakey Clinical Medical Research Award recognizes the contributions of Ralf Bartenschlager, Charles Rice, and Michael Sofia (Figure 1) toward the development of HCV cell culture systems and the exploitation of these systems to develop drugs to target this lethal virus.

\section{An unknown source of progressive hepatitis}

In the 1970s, diagnostic tools became available to identify both hepatitis A virus
(HAV) and hepatitis B virus (HBV) infections in patients that had developed blood transfusion-associated hepatitis as well as in donated blood prior to use in transfusions. It quickly became clear that the majority of patients screened were not infected with either of these viruses and ated from DNA and RNA isolated from the pellet of ultracentrifuged plasma of a NANBH-infected chimpanzee. Only one of more than one million of clones in this library expressed an antigen that bound antibodies collected from NANBHinfected patients and chimpanzees. Addi-
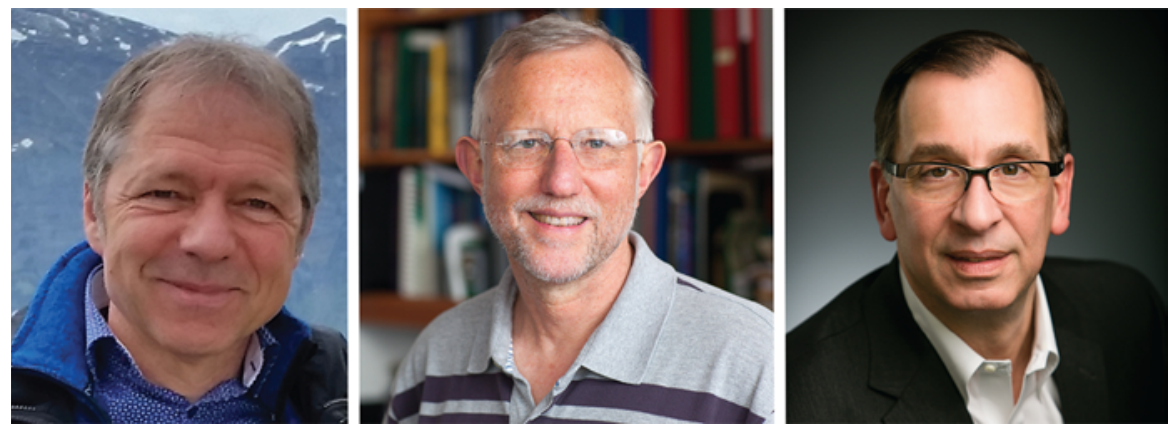

Figure 1. Ralf Bartenschlager (left), Charles Rice (center), and Michael Sofia (right) are the recipients of the $\mathbf{2 0 1 6}$ Lasker DeBakey Clinical Medical Research Award. Image credit for photo of Charles Rice: John Abbot/The Rockefeller University.

that some unknown agent, which was termed NANBH, was responsible. There was an intensive effort from many groups to identify and characterize the NANBH agent; however, the agent proved intractable to conventional immunological techniques and in vitro propagation in tissue culture. Moreover, the underlying cause of NANBH was not easily traced, as antibodies in the sera of individuals or nonhuman primates with NANBH could not reliably detect antigen in infected tissues (see ref. 2 for a comprehensive review of these efforts). In 1989, a group led by Michael Houghton at the biotechnology company Chiron published a study identifying $\mathrm{HCV}$ as the agent responsible for NANBH (3). Using sera collected from a NANBH-infected patient with severe liver disease, they screened a bacterial cDNA expression library that had been gener- tionally, this clone allowed a nearly complete determination of the HCV genome sequence, revealing sequence homology to flaviviruses, such as yellow fever virus. The identification of HCV quickly led to the development of assays for detection of HCV, allowing the identification and exclusion of potentially infected blood. Moreover, these studies quickly led to the realization that HCV was a major cause of hepatitis throughout the world (4).

\section{The long road toward cell- based systems}

The identification of HCV was a huge advance for the field, leading to the identification of different HCV genotypes and subtypes based on genetic sequence differences. While much information was gleaned from studying the HCV genome, many aspects of the disease, including the viral life cycle, requirements for host cell entry, and the viral components with potential as drug targets, were not readily inves- 
HCV RNA
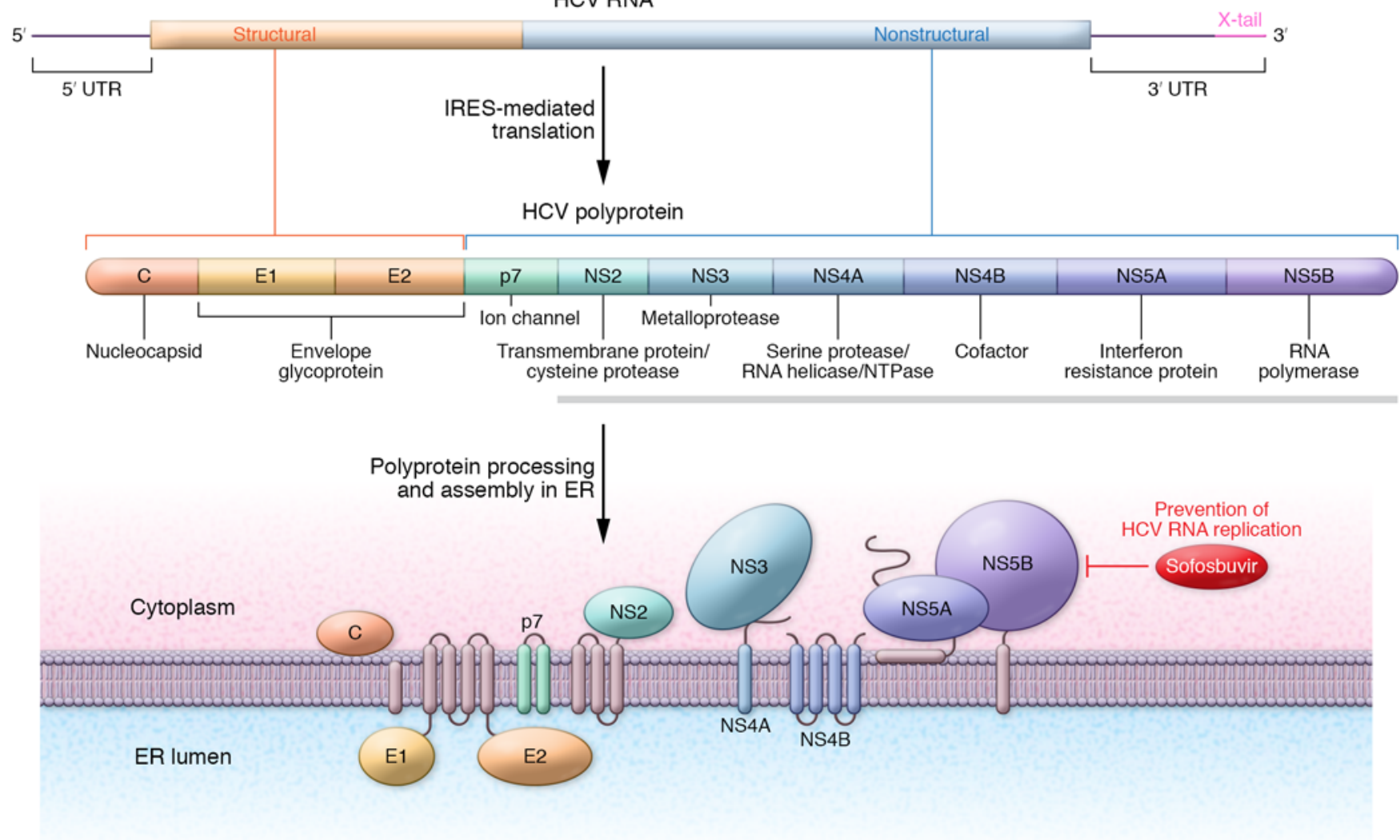

Figure 2. Schematic representation of translation and assembly of HCV proteins in the host ER. The HCV RNA transcript encodes both structural and nonstructural HCV proteins. The untranslated regions (UTR) at the $5^{\prime}$ and $3^{\prime}$ ends are essential, as they contain sequence and structural elements that are critical for HCV translation and RNA replication. Initial HCV genome sequences did not include all of the 3' UTR, and it was later shown that the $3^{\prime}$ X-tail of the HCV transcript is essential for viral production. HCV is translated as a polyprotein that contains both structural and nonstructural proteins. Subgenomic replicons containing the HCV replication machinery (gray bar) have been invaluable for studies of the HCV replication. The HCV polyprotein undergoes photolytic processing and is assembled in the host ER. Several HCV proteins have been explored as drug targets, including the viral RNA polymerase (NS5B). Sofosbuvir, which targets the viral RNA polymerase (NS5B), was approved by the FDA in 2013 and is associated with very high cure rates.

tigated. Around this time two researchers, Charles Rice and Ralf Bartenschlager, became involved in the study of $\mathrm{HCV}$; these individuals would be instrumental in the development of the necessary tools for understanding this lethal virus.

In the US, Charles Rice, an assistant professor at Washington University in St. Louis, had just published a paper describing the development of an in vitro system that allowed functional generation of the yellow fever vaccine strain (17D) from cDNA (5). Rice had an extensive background in virology, especially in evaluating genome structure and sequence, having done his dissertation and a postdoctoral stint with Jim and Ellen Strauss at the California Institute of Technology. As Rice recently recounted to the JCI of his experience in the Strauss lab, "I really enjoyed working on viruses in that environment. I was given a lot of freedom to pursue some good ideas and some ideas that were maybe before their time - or more challenging than my abilities at the time." Based on his experience with flaviviruses, Rice was on the radar of Stephen Feinstone, who worked on viral hepatitis at the FDA and was interested in collaborating with Rice. According to Rice, "[at this time], we knew the majority of the genome sequence, but we didn't really know where the individual virus-encoded proteins started and stopped."

In Germany, Ralf Bartenschlager first became interested in viruses during his time as a graduate student and postdoc at the University of Heidelberg, where he studied under molecular virologist Heinz Schaller. In particular, his research focused on structural and functional evaluation of HBV proteins. In 1991, Bartenschlager accepted a position at Hoffmann-La Roche in Basel, Switzerland, where he was tasked with developing an $\mathrm{HCV}$ research program for the company. Several years after he started this program, Bartenschlager was informed that his branch of the company would be leaving Basel to move to the UK. Without a guarantee that he would be able to return to Switzerland, Bartenschlager took a position at the University of Mainz where he continued his research on HCV. Like many others in the field, he was particularly interested in finding a way to culture the virus in vitro. Bartenschlager recently discussed his initial work at the University of Mainz with the JCI: "At the University of Mainz, we mainly worked on the followup of the genome sequence, looking at the genomic organization as well as determining the viral proteins, how they are made, and what is their enzymatic activity. We pretty much studied anything that you could evaluate in a test tube, but we knew we were coming to a dead end very soon if we did not have a culture system." 
During the 1990s, several insights into $\mathrm{HCV}$ were gained by studying the genome and infection in nonhuman primates; however, HCV could not undergo replication in existing cell culture systems. With genome sequences available, attempts to develop transfection-based replication strategies began. Such an approach involves bacteriophage promoter-driven synthesis of the viral RNA genome using a recombinant DNA plasmid template. The transcribed viral genome is then purified and transfected into mammalian cells, in which it should be recognized and translated by the host machinery. This approach has been successful for many other positive-strand RNA viruses (6), but HCV would again resist these initial attempts. Unfortunately, initial constructs were based on incomplete genomes that lacked part of the $3^{\prime}$ UTR, and these genomes were unable to infect chimpanzees. In 1995 and 1996, two teams, one led by Charles Rice and the other by Kunitada Shimotohno, independently identified a unique sequence downstream of what had been considered the $3^{\prime}$ end of the viral RNA. Called the X-tail (Figure 2), it had no homology to any known viral or nonviral sequences, was highly conserved among HCV subtypes, and was predicted to form a stemloop structure that is essential for proper recruitment of the replication machinery to the viral transcript (7-9).

With the identification of the X-tail and further characterization of HCV consensus sequences, Rice and his colleagues would go on to generate the first infectious HCV clones. The group, spearheaded by Alexander (Sasha) Kolykhalov, generated 10 full-length clone derivatives based on consensus sequences of HCV genotype 1a strain $\mathrm{H} 77$, which was originally isolated from a patient with acute-stage infection. Transcripts from these 10 clones were pooled and delivered by intrahepatic injection to two naive chimpanzees. Both animals had measurable viremia and evident hepatitis (10). Less than a month later, a group led by Jens Bukh would publish its work, which also showed that RNA transcripts generated from cDNA clones of the consensus H77 genome (with the $\mathrm{X}$-tail) were infectious in a chimpanzee (11). Despite successfully infecting chimpanzees, these clones were unable to replicate or produce $\mathrm{HCV}$ in cultured cells. As
Rice recalls, "Attempts to use these chimpanzee-infectious RNA transcripts to find evidence of replication in cell culture were met with uniform failure in our lab and others. We had finally overcome one barrier and then ran right into another roadblock. That's where Ralf Bartenschlager and Volker Lohmann came in.'

\section{Selection under pressure}

In the 1990s, work in Bartenschlager's lab was focused on the development of a transfection-based cell culture model. Bartenschlager had a very dedicated $\mathrm{PhD}$ student in the lab at this time, Volker Lohmann. According to Bartenschlager, "He spent a lot of time trying to set up the cell culture system - probably the first three years of his thesis. At that point we all said he should do something else, because otherwise he was at risk of having a thesis without any data." Lohmann did take a break and focused on the HCV RNA-dependent RNA polymerase NS5B for his thesis project $(12,13)$. After successfully defending his thesis, Lohmann continued with Bartenschlager and went back to trying to develop an HCV cell culture system. The Bartenschlager group had carefully generated virus-encoding constructs based on the consensus sequence of the HCV genotype $1 \mathrm{~b}$ strain they called Con1, making sure the complete 3 ' UTR of the viral genome was present. Moreover, his group had developed methods to make sure that all traces of plasmid DNA were removed from their preparations and they utilized highly sensitive and specific quantitative PCR methods. Despite this meticulous approach, they were unable to convincingly detect viral replication, leading them to try other methods.

Studies of poliovirus had shown that structural proteins are not required for viral replication in cells (14), giving the Bartenschlager group the encouragement they needed for their next attempt, which focused on the identity of cells permissible to replication. They generated subgenomic replicons in which the structural genes of HCV Con1 were replaced with a selectable marker. They also inserted an internal ribosome entry site (IRES) from encephalomyocarditis virus (EMCV), which is able to initiate translation in a variety of cell types upstream of the genes encoding the assumed HCV replication machinery.
Thus, the HCV replication machinery is translated from the EMCV IRES, while the selectable marker, in this case neomycin phosphotransferase (npt, which confers resistance to the cytotoxic drug G418), is synthesized from the native HCV IRES. Only transfected cells that are able to support HCV replication would survive treatment with G148. This approach revealed that the human hepatoma cell line Huh-7 could support HCV replication (15). Though only a small number of transfected Huh-7 cells supported replication, those that did had high levels of HCV RNA replication.

The HCV replicon system would be further improved by the identification of mutations that enhanced replication. These mutations most frequently occur in NS5A, followed by NS3, and NS4B (16-19) (Figure 2). Additionally, mutations within Huh-7 cells were identified that improved the ability of the cells to replicate HCV replicons $(20,21)$. The identification of both viral and host cell mutations that allowed for a highly permissive environment would pave the way for the development of replicons representing all HCV genotypes and for the establishment of other cell lines that support HCV replication (22). There was excitement with the establishment of these highly permissible replicon systems; it was initially thought that simply adding back the structural components of the HCV genome would be enough to establish infectious particles. However, as Charles Rice told the JCI, "HCV had other ideas." Jens Bukh, together with Bartenschlager's team, showed that in vitro-generated transcripts derived from constructs with the consensus HCV strain Con1 genome sequence were infectious when intrahepatically injected into chimpanzees; however, in vitro-derived transcripts from constructs containing the Con 1 genome with replication-efficient mutations were not infectious (23).

\section{A unique isolate brings HCV full cycle}

The search was on for an HCV isolate that would be able to replicate efficiently in Huh-7 cells without replication-enhancing mutations. The strain that would meet these criteria was isolated in 2003 , by a group led by Takaji Wakita, from a Japanese patient with fulminant hepatitis, a relatively rare occurrence in newly infected individu- 
als (24). This isolate, JFH-1, is a genotype 2a strain, and Wakita and colleagues found that subgenomic replicons of this strain replicated well in Huh-7 cells without the introduction of replication-enhancing mutations (24). Wakita shared his isolate with others in the field, and in 2005 , roughly 15 years after the identification of $\mathrm{HCV}$, three independent teams led by Wakita in collaboration with Ralf Bartenschlager, Charles Rice, and Francis Chisari reported the complete replication of $\mathrm{HCV}$ in cell culture and production of infectious particles from constructs based on the JFH-1 sequence (25-27). As Bartenschlager recently told the JCI, "The JFH-1 virus was the jackpot. For reasons we still don't know, this isolate replicates extremely efficiently and does not require mutations." Rice concurred that the JFH-1 virus is special: "It was really an amazing breakthrough because constructs made with this virus could be shown to be infectious in naive cultured cells, infectious in the chimpanzee model, and infectious in humanized mice." Finally, the full life cycle of HCV could be studied, leading to a better understanding of the host and viral factors required for replication and viral entry into host cells (for reviews, see refs. 22, 28, 29).

\section{Searching for a better cure}

Since 1990, the incidence of HCV has been on the rise, with the WHO estimating that approximately $2 \%$ of the global population was infected with HCV in 2004 (30, 31). Accordingly, cirrhosis-, liver disease-, and hepatic cancer-related deaths have increased and remain a leading cause of death worldwide. IFN-based therapies were being explored to treat patients with viral hepatitis prior to the identification of $\mathrm{HCV}$ as the causative agent of NANBH (32), and they remained an integral part of the standard of care until the 2010s, especially in combination with ribavirin (RBV) (33). While this course of treatment was able to induce a sustained virologic response rate in approximately $42 \%$ of patients after 48 weeks of treatment, the side effects, including fatigue, flu-like symptoms, headache, insomnia, and hematological abnormalities, such as anemia and neutropenia, caused many patients to abandon treatment (34). The development and improvement of both the replicon system and the JFH-1-based culture systems provided a platform to evaluate potential HCV drugs for efficacy against multiple HCV subtypes.

Michael Sofia became interested in the interface between biology and chemistry as a high school student in Baltimore, Maryland, and his attraction to this field increased during his time as an undergraduate at Cornell University. His undergraduate research advisor arranged a paid summer internship for him at Johns Hopkins University School of Medicine with Cecil Robinson, as it was not feasible for Sofia to stay in Ithaca, New York, over the summer break. As Sofia recently told the JCI, "It was one of those situations where the switch went on. Cecil Robinson was doing work that involved designing molecules to inhibit enzymes related to a biological pathway that would be implicated in breast cancer. This was the first exposure I had to designing a molecule to impact a biological problem." Sofia would go on to graduate school at the University of Illinois, followed by a postdoctoral stint at Columbia University to hone his skills in chemical synthesis, before going into the pharmaceutical industry. Sofia joined the small biotech company Pharmasset in 2005 to lead their chemistry research effort. At the time, the company's HCV program was in its infancy, but Sofia saw potential in the compounds they were developing. "The company had identified a nucleoside that had activity against HCV replication in the replicon assay. It had interesting activity but had issues that made it a poor drug candidate .... It also had other characteristics that made it interesting to pursue, including the fact that the molecule was specific for HCV versus other viruses, it was able to inhibit the polymerases generated from multiple $\mathrm{HCV}$ genotypes, and had potential to have a high barrier to resistance." This molecule (PSI-6130) targets the HCV NS5B RNA polymerase (Figure 2) and thereby prevents elongation of the viral RNA genome (35). This molecule is part of a class of drugs known as direct acting antivirals, which target the virus as opposed to boosting the host immune response; thus, these drugs should have fewer adverse side effects.

The Pharmasset team would spend the next few years developing and testing different variations of their original compound, with the goal of increasing the halflife, potency, and liver-selective delivery of the active drug, but they were not able to achieve these objectives. The team then went back to the drawing board and redesigned the molecule, keeping some features of the original compound but adding several new ones drawing on information they developed on how the body metabolized the original compound. For Sofia, this meant utilizing hepatic metabolic pathways to develop a prodrug that would be unmasked and activated in the liver. These efforts cumulated with the generation of PSI-7851, which showed specificity for $\mathrm{HCV}$, pan-recognition of HCV genotypes, and low cytotoxicity (36). Moreover, PSI-7851 cleared HCV replicons from transfected cells, with no viral rebound after drug removal (37). In clinical safety and tolerability trials, PSI-7851 was shown to be safe and to have a pharmacokinetic profile favorable for once-a-day dosing and a decrease in plasma HCV RNA after 3 days of dosing in treatment-naive patients. With clinical proof of concept for the drug design now achieved, one more thing needed to be done. PSI-7851 was actually a mixture of two forms of the molecule, in which one form was more potent than the other. Isolation and preparation of the more active form was achieved, and this molecule, PSI-7977, became known as sofosbuvir. Sofosbuvir quickly moved into phase II clinical testing, first with IFN/RBV, with which it showed exceptional potency in a 28-day study; however, it wasn't until a clinical study called ELECTRON that the full potential of sofosbuvir was realized. The ELECTRON trial evaluated an IFN-free combination with RBV and produced a $100 \%$ cure rate in a genotype 2 and 3 patient cohort, thus forever changing the treatment of HCV patients $(38,39)$. Subsequently, phase III trials led to the regulatory approval of sofosbuvir, either in combination with IFN for genotype 1 and 4 patients or in combination only with RBV for genotype 2 and 3 patients as the first IFN-free treatment regimen $(40,41)$. Later combinations of sofosbuvir with the NS5A inhibitor ledipasvir provided an IFN-free regimen that produced a $>95 \%$ cure rate in genotype 1 patients (42). Today, sofosbuvir serves as the backbone for HCV therapy. As Sofia recently remarked, "It is the backbone, because it has this great resistance profile and it has pan-genotypic characteristics, which none of the other agents 
can really provide. It really is amazing: all patient populations can take it, whether they are genotype 1 or 6 ; whether they are cirrhotic or noncirrhotic; whether their previous treatment regimens have failed or not." Importantly, over $90 \%$ of patients that complete a 12-week course of sofosbuvir treatment will be clear of the infection, with few, if any, adverse effects.

\section{Looking to the future}

The HCV field has come a long way since the identification of the virus in 1989. As Sofia told the JCI, "I think that we are now on the verge of being able to eradicate HCV. One pill, once a day for 8-12 weeks, and you can clear HCV." For Charles Rice, there is still much to be learned about the basic biology of HCV. "We still haven't answered a lot of basic questions about the mechanisms used by this virus to effectively establish these chronic infections. How does chronic infection in some people give rise to a fairly benign outcome in terms of liver disease progression, whereas, in other people, there is a progression to advanced fibrosis, cirrhosis, and cancer? We don't really understand those mechanisms, we don't understand whether or not the mechanism of liver cancer development is a direct viral effect or just an effect of prolonged inflammation in the liver leading to cellular turnover and division. There are really a lot of aspects about the immunology and pathogenesis that we have not managed to solve." Bartenschlager told the JCI that there are issues facing HCV eradication: "Drugs need to be available for all infected populations, not everyone can afford them. Additionally, there still is a relatively high number of undiagnosed people. A prophylactic vaccine should not be forgotten when discussing global elimination of $\mathrm{HCV}$."

Globally, viral hepatitis is on the rise, and the number deaths per year as the result of virus-induced cirrhosis and liver cancer is now on par with the number of deaths associated with HIV and tuberculosis (43). In 2016, the WHO released its first global health sector strategy on viral hepatitis, with the goal of eliminating viral hepatitis as a public health threat by 2030, particularly those cases caused by HBV and HCV (43). This ambitious plan would not be possible without the work of Ralf Bartenschlager, Charles Rice, and Michael Sofia. The 2016
Lasker DeBakey Clinical Medical Research Award honors these three individuals who have truly demonstrated how basic scientific discoveries can pave the way for developing cures for disease.

\section{Corinne L. Williams}

1. Hepatitis C Fact Sheet. World Health Organization. http://www.who.int/mediacentre/factsheets/fs164/en. Updated July 2016. Accessed August 23, 2016.

2. Houghton $M$. The long and winding road leading to the identification of the hepatitis $C$ virus. JHepatol. 2009;51(5):939-948.

3. Farci P. Choo QL, Kuo G, Weiner AJ, Overby LR, Bradley DW, Houghton M. Isolation of a cDNA clone derived from a blood-borne non-A, non-B viral hepatitis genome [Science 1989;244:359362]. J Hepatol. 2002;36(5):582-585.

4. Kuo G, et al. An assay for circulating antibodies to a major etiologic virus of human non-A, non-B hepatitis. Science. 1989;244(4902):362-364.

5. Rice CM, Grakoui A, Galler R, Chambers TJ. Transcription of infectious yellow fever RNA from full-length cDNA templates produced by in vitro ligation. New Biol. 1989;1(3):285-296.

6. Boyer JC, Haenni AL. Infectious transcripts and cDNA clones of RNA viruses. Virology. 1994;198(2):415-426.

7. Tanaka T, Kato N, Cho MJ, Shimotohno K. A novel sequence found at the 3 ' terminus of hepatitis $\mathrm{C}$ virus genome. Biochem Biophys Res Commun. 1995;215(2):744-749.

8. Kolykhalov AA, Feinstone SM, Rice CM. Identification of a highly conserved sequence element at the 3' terminus of hepatitis $C$ virus genome RNA. JVirol. 1996;70(6):3363-3371.

9. Tanaka T, Kato N, Cho MJ, Sugiyama K, Shimotohno K. Structure of the 3' terminus of the hepatitis C virus genome. J Virol.1996;70(5):3307-3312.

10. Kolykhalov AA, Agapov EV, Blight KJ, Mihalik $\mathrm{K}$, Feinstone SM, Rice CM. Transmission of hepatitis $\mathrm{C}$ by intrahepatic inoculation with transcribed RNA. Science. 1997;277(5325):570-574.

11. Yanagi M, Purcell RH, Emerson SU, Bukh J. Transcripts from a single full-length cDNA clone of hepatitis $\mathrm{C}$ virus are infectious when directly transfected into the liver of a chimpanzee. Proc Natl Acad Sci U S A. 1997;94(16):8738-8743.

12. Lohmann V, Roos A, Körner F, Koch JO, Bartenschlager R. Biochemical and kinetic analyses of NS5B RNA-dependent RNA polymerase of the hepatitis C virus. Virology. 1998;249(1):108-118.

13. Lohmann V, Körner F, Herian U, Bartenschlager R. Biochemical properties of hepatitis $C$ virus NS5B RNA-dependent RNA polymerase and identification of amino acid sequence motifs essential for enzymatic activity. J Virol. 1997;71(11):8416-8428.

14. Kaplan G, Racaniello VR. Construction and characterization of poliovirus subgenomic replicons. JVirol. 1988;62(5):1687-1696.

15. Lohmann V, Körner F, Koch J, Herian U, Theilmann L, Bartenschlager R. Replication of subge- nomic hepatitis $C$ virus RNAs in a hepatoma cell line. Science. 1999;285(5424):110-113.

16. Blight KJ, McKeating JA, Marcotrigiano J, Rice CM. Efficient replication of hepatitis $C$ virus genotype 1a RNAs in cell culture. JVirol. 2003;77(5):3181-3190.

17. Blight KJ, Kolykhalov AA, Rice CM. Efficient initiation of HCV RNA replication in cell culture. Science. 2000;290(5498):1972-1974.

18. Lohmann V, Körner F, Dobierzewska A, Bartenschlager R. Mutations in hepatitis $\mathrm{C}$ virus RNAs conferring cell culture adaptation. J Virol. 2001;75(3):1437-1449.

19. Krieger N, Lohmann V, Bartenschlager R. Enhancement of hepatitis $\mathrm{C}$ virus RNA replication by cell culture-adaptive mutations. J Virol. 2001;75(10):4614-4624.

20. Murray EM, et al. Persistent replication of hepatitis $\mathrm{C}$ virus replicons expressing the beta-lactamase reporter in subpopulations of highly permissive Huh7 cells. JVirol. 2003;77(5):2928-2935.

21. Blight KJ, McKeating JA, Rice CM. Highly permissive cell lines for subgenomic and genomic hepatitis C virus RNA replication. J Virol. 2002;76(24):13001-13014.

22. Catanese MT, Dorner M. Advances in experimental systems to study hepatitis $C$ virus in vitro and in vivo. Virology. 2015;479-480:221-233.

23. Bukh J, et al. Mutations that permit efficient replication of hepatitis $C$ virus RNA in Huh-7 cells prevent productive replication in chimpanzees. Proc Natl Acad Sci U S A. 2002;99(22):14416-14421.

24. Kato T, et al. Efficient replication of the genotype 2a hepatitis $C$ virus subgenomic replicon. Gastroenterology. 2003;125(6):1808-1817.

25. Wakita T, et al. Production of infectious hepatitis $C$ virus in tissue culture from a cloned viral genome. Nat Med. 2005;11(7):791-796.

26. Zhong J, et al. Robust hepatitis $C$ virus infection in vitro. Proc Natl Acad Sci U S A. 2005;102(26):9294-9299.

27. Lindenbach $\mathrm{BD}$, et al. Complete replication of hepatitis $C$ virus in cell culture. Science. 2005;309(5734):623-626.

28. Scheel TK, Rice CM. Understanding the hepatitis $\mathrm{C}$ virus life cycle paves the way for highly effective therapies. Nat Med.2013;19(7):837-849.

29. Lohmann V, Bartenschlager R. On the history of hepatitis $\mathrm{C}$ virus cell culture systems. JMed Chem. 2014;57(5):1627-1642.

30. Stanaway JD, et al. The global burden of viral hepatitis from 1990 to 2013: findings from the Global Burden of Disease Study 2013. Lancet. 2016;388(10049):1081-1088.

31. Global Burden Of Hepatitis $C$ Working Group. Global burden of disease (GBD) for hepatitis C. JClin Pharmacol. 2004;44(1):20-29.

32. Hoofnagle JH, et al. Treatment of chronic non-A, non-B hepatitis with recombinant human alpha interferon. A preliminary report. $N$ Engl J Med. 1986;315(25):1575-1578.

33. Strader DB, Seeff LB. A brief history of the treatment of viral hepatitis C. Clin Liver Dis. 2012;1:6-11.

34. Fried MW. Side effects of therapy of hepatitis $C$ and their management. Hepatology. 2002; 36(5 Suppl 1):S237-S244.

35. Clark JL, et al. Design, synthesis, and antiviral activity of 2'-deoxy-2'-fluoro-2'-C-methylcyti- 
dine, a potent inhibitor of hepatitis $\mathrm{C}$ virus replication. J Med Chem. 2005;48(17):5504-5508.

36. Sofia MJ, et al. Discovery of a $\beta-d-2$ '-deoxy-2' $-\alpha$ fluoro-2'- $\beta$-C-methyluridine nucleotide prodrug (PSI-7977) for the treatment of hepatitis $\mathrm{C}$ virus. JMed Chem. 2010;53(19):7202-7218.

37. Lam AM, et al. PSI-7851, a pronucleotide of beta-D-2'-deoxy-2'-fluoro-2'-C-methyluridine monophosphate, is a potent and pan-genotype inhibitor of hepatitis $\mathrm{C}$ virus replication. Antimicrob Agents Chemother. 2010;54(8):3187-3196.

38. Gane E, et al. Once Daily GS-7977 Plus Ribavirin in HCV Genotypes 1-3: The ELECTRON Trial.
Paper presented at 47th Annual Meeting of the European Association for the Study of the Liver: 2012. http://www.natap.org/2012/EASL/ EASL_33.htm. Accessed August 25, 2016.

39. Gane EJ, et al. 14 all-oral sofosbuvir-based 12-week regimens for the treatment of chronic HCV infection: the ELECTRON study. JHepatol. 2013;58:S6-S7.

40. Lawitz E, et al. Sofosbuvir for previously untreated chronic hepatitis C infection. $N$ EnglJ Med. 2013;368(20):1878-1887.

41. Jacobson IM, et al. Sofosbuvir for hepatitis C genotype 2 or 3 in patients without treatment options. N Engl J Med.2013;368(20):1867-1877.

42. Lawitz E, et al. Sofosbuvir and ledipasvir fixed-dose combination with and without ribavirin in treatment-naive and previously treated patients with genotype 1 hepatitis $C$ virus infection (LONESTAR): an openlabel, randomised, phase 2 trial. Lancet. 2014;383(9916):515-523.

43. Global Health Sector Strategy on Viral Hepatitis 2016-2021. World Health Organization. http:// apps.who.int/iris/bitstream/10665/246177/1/ WHO-HIV-2016.06-eng.pdf. Published June 2016. Accessed August 23, 2016 